\title{
A Simple Approach to Analyze Sugar Nectar Composition in Flowers Using Capillary Electrophoresis and Enzymatic Assays
}

\author{
Dayanne M. Bordin, ${ }^{a}$ Samara G. C. Latgé, ${ }^{b}$ Graham Pyke, ${ }^{c}$ John Kalman, ${ }^{a}$ Philip Doble, ${ }^{a}$ \\ Fernando A. Genta ${ }^{c}$ and Lucas Blanes ${ }^{\odot * d}$
}

\author{
${ }^{a}$ School of Mathematical and Physical Sciences, University of Technology Sydney, Ultimo, \\ 2007 New South Wales, Australia \\ ${ }^{b}$ Laboratório de Bioquímica e Fisiologia de Insetos, Fundação Oswaldo Cruz (Fiocruz), \\ 21040-360 Rio de Janeiro-RJ, Brazil
}
'Department of Biological Sciences, Macquarie University, Ryde, 2109 New South Wales, Australia
${ }^{d}$ Laboratório de Ciências e Tecnologias Aplicadas em Saúde, Instituto Carlos Chagas, Fundação Oswaldo Cruz (Fiocruz), 81350-010 Curitiba-PR, Brazil

\begin{abstract}
Despite the obvious importance of nectar, the composition of this solution remains surprisingly understudied to most of the flowers. Here we describe a simple, low-cost, and reliable methodology to analyze the three main sugars present in 210 nectar samples of Christmas Bellflowers using capillary electrophoresis (CE) and enzymatic assays. CE separation $(-16 \mathrm{kV})$ was made using an electrolyte containing $36 \mathrm{mM} \mathrm{NaOH} \mathrm{pH} \mathrm{12,} 15 \mathrm{mM}$ sorbic acid, $0.5 \mathrm{mM}$ hexadecyl trimethylammonium bromide (CTAB), and indirect UV detection. $72.9 \%$ of analyzed nectar samples were sucrose dominant; $13.3 \%$ sucrose rich only and $13.8 \%$ were fructose rich. The concentrations of fructose and glucose present in most of the samples were very similar, while the amount of sucrose varied considerably. The average amounts of fructose, glucose, sucrose and total sugar were $0.22,0.21,0.34$ and $0.8 \mathrm{M}$, respectively. The nectar sample shows a $\mathrm{pH}$ average of 5 . $\alpha$-Glucosidase activity was observed in $65 \%$ of the samples with an average activity of $0.013 \mu \mathrm{U} \mu \mathrm{L}^{-1}$ providing a possible explanation for the similar concentrations of fructose and glucose in the samples. Under our knowledge, this is the first article that shows the analysis of sugars in nectar flowers using CE.
\end{abstract}

Keywords: capillary electrophoresis, nectar, sucrose, glucose, fructose

\section{Introduction}

The process of pollination is essential for life on Earth, and approximately $90 \%$ of all flowering plant species are animal pollinated. Floral nectar is an important reward to animals to facilitate pollination, however, floral nectars remain surprisingly understudied. ${ }^{1,2}$ Nectar components, including sugars, amino acids, and other classes of substances are not well characterized and new compounds continue to be discovered. ${ }^{3}$ Nectars mainly contain varying proportions of glucose, sucrose, fructose and with smaller quantities of other sugars and organic and inorganic substances. ${ }^{4}$

Nectar characteristics such as concentration, sugar composition, time of nectar secretion, sucrose-hexose

\footnotetext{
*e-mail: Lucas.Blanes@ fiocruz.br
}

proportions, volume and nectar dynamics are regularly related to the interaction of flowers and pollinators, with correlations or 'syndromes' between nectar and pollinator attributes. ${ }^{5}$ The homogeneity of nectar sugar is shown in the literature as a conservative feature fairly constant within taxonomically related species. In order to determine the nectar features role in the interactions between plants and flower visitors, field observations and mathematical modelling are required. ${ }^{6}$

Glucose and fructose do not necessarily occur in similar amounts in floral nectar, as they are partly and differentially recycled through various biochemical pathways before being secreted. ${ }^{4}$ Invertase enzymes, such as transglucosidase and transfructosidase, have been identified in the secretory mechanism, showing essential functions of nectar chemistry. ${ }^{1} \alpha$-Glucosidases may also 
play an important role on nectar biochemistry since these enzymes cleave the main disaccharides found in nectar. ${ }^{7}$

Chromatographic methods are the most used analytical techniques for the analysis of sugars in nectar. As the concentration of sugars in nectar are relatively high, samples, in general, need to be diluted before analysis, therefore sensitivity is not an issue. Sugars can be analyzed by thin-layer chromatography (however this technique does not precisely quantify these analytes) or highperformance liquid chromatography (HPLC), which uses high amounts of solvents and expensive columns. ${ }^{8-11} \mathrm{Gas}$ chromatography (GC) also can be used, but in general, also requires laborious derivatization procedures. ${ }^{12}$ Since direct UV absorption of sugars is limited to 190 to $195 \mathrm{~nm}, \mathrm{UV}$ detection is practical only if uncontaminated sample and water as mobile phase are applied. Sugars analyzes are also widely performed by HPLC coupled with refractive index detection. This detection method is considered standard for sugars, however its selectivity is poor. Slight variations in the mobile phase concentrations and temperature are associated to changes in the refractive index; in addition, literature ${ }^{9}$ shows that methods using borate complex anion exchange, appear to present boric acid system peaks as sugar peaks. HPLC coupled with fluorescence detection also is an alternative, however considerable time to be derivatized is required, and fluorescence sensors are also expensive. HPLC coupled with electrochemical detection also is an alternative, however it presents low detection selectivity to target components. ${ }^{9,10,13}$

In capillary electrophoresis $(\mathrm{CE})$, the analytes migrate through a capillary column filled with an electrolyte solution due to the influence of an electric field. CE is a powerful tool that can be used for the analysis of charged and uncharged species, including sugars. This technique presents several advantages in comparison with other analytical techniques such as small sample and reagent volumes, fast analysis time, do not require the use of expensive columns, solvents or gases, it is a high-resolution technique, and produce minimal waste..$^{9,14-20}$ There are some articles described in the literature ${ }^{9,14}$ showing the analysis of different substances in plant extracts via $\mathrm{CE}$, including sugars, however none of them in nectar. One of the strategies to analyze neutral sugars by CE is to use highly alkaline electrolytes with a $\mathrm{pH}$ value higher than 12 to ionize the hydroxyl groups ( $\mathrm{p} K_{\mathrm{a}} \mathrm{ca} .12$ ), converting them in anionic species. As carbohydrates lack absorption in the UV region, indirect detection is commonly used by adding an absorbing agent such as sorbic acid into the background electrolyte. Sorbic acid is an ideal probe for the analysis of sugars in CE as its mobility is similar to fructose, glucose and sucrose, thereby minimizing dispersion and providing excellent peak symmetries. ${ }^{9}$ In this article we present the validation of a $\mathrm{CE}$ method for the analysis of sucrose, glucose and fructose in 210 nectar samples of Christmas Bellflowers (Blandfordia grandiflora) as well as the biochemical detection of an $\alpha$-glucosidase activity on the samples. Under our knowledge, this is the first article that shows the analysis of sugars in nectar flowers using CE.

\section{Experimental}

\section{Reagents and chemicals}

Sorbic acid was acquired from BDH Laboratory Chemicals Division (Poole, England). Hexadecyl trimethylammonium bromide (CTAB) was purchased from Sigma-Aldrich (Castle Hill, Australia). Sodium hydroxide $(\mathrm{NaOH})$ was obtained from Chem-supply (Adelaide, Australia), glucose anhydrous from Analytical Univar reagent (Sydney, Australia), D-fructose from M\&B Laboratory Chemicals May and Baker LTD (Dagenham, England). Sucrose and galactose were obtained from Mallinckrodt AR, Lab Guard (Dublin, Ireland). The reagents 4-methylumbelliferyl- $\alpha$-D-glucopyranoside (MU $\alpha \mathrm{Glu}$ ) and 4-methylumbelliferone were purchased from Sigma-Aldrich Company (St. Louis, Missouri, USA). Glucose oxidase-based was purchased from Bioclin (Belo Horizonte, Minas Gerais, Brazil). Analytical grade reagents and purified water were used (Arium ${ }^{\circledR}$ Pro-Sartorius, Melbourne, Australia). $100 \mathrm{mM}$ stock solutions of sucrose, fructose and glucose were prepared in ultra-pure water and stored at $4{ }^{\circ} \mathrm{C}$ and diluted accordingly to give the required concentrations.

\section{Instrumentation and electrophoretic procedures}

Capillary electrophoresis (CE) analyses were performed on a CE system (model 7100, Agilent Technologies, Palo Alto, CA, USA), equipped with a diode array detector set at 190 to $600 \mathrm{~nm}$. The data were processed using the software HP ChemStation supplied by the manufacture. Samples were injected by pressure (50 mbar for $8 \mathrm{~s}$ ) in a $70 \mathrm{~cm}$ fused-silica capillary (50 $\mu \mathrm{m}$ inner and $360 \mu \mathrm{m}$ outer diameter), $65 \mathrm{~cm}$ effective length, from Polymicro Technologies, Phoenix, (AZ, USA). The capillary temperature was maintained at $25^{\circ} \mathrm{C}$ and the separation voltage was $-16 \mathrm{kV}$.

The electrolyte solution was at $\mathrm{pH} 12$, prepared using $15 \mathrm{mM}$ sorbic acid, $0.5 \mathrm{mM} \mathrm{CTAB}$ and $36 \mathrm{mM} \mathrm{NaOH}$. Prior to analysis, the capillary was conditioned by flushing it with $1 \mathrm{M} \mathrm{NaOH}$ (15 min) followed by a flush of deionized water and electrolyte solution, for 2 and $15 \mathrm{~min}$, respectively. 
The capillary was flushed for 2 min between runs with the electrolyte. At the end of each working day, the capillary was rinsed with water for $5 \mathrm{~min}$ in order to avoid any residue of $\mathrm{NaOH}$ that could damage the capillary walls. A diodearray detector was used for indirect UV monitoring. The signal wavelength was set at $254 \mathrm{~nm}$ with a bandwidth of $80 \mathrm{~nm}$ and the reference was at $450 \mathrm{~nm}$ with a bandwidth of $80 \mathrm{~nm}$.

Method validation

During the validation process, the limit of detection (LOD), the limit of quantification (LOQ), linearity, precision, and accuracy were evaluated. The linearity was measured using six calibration points in triplicate, in three independent runs. The concentrations of calibration points were: $0.1,0.25,0.50,1,2$ and $4 \mathrm{mM}$ for fructose, glucose, and sucrose. Galactose was used as an internal standard (IS) at a final concentration of $1 \mathrm{mM}$. Coefficient of determination $\left(\mathrm{R}^{2}\right)$ and least square regression line were used to express and evaluate the linearity response. All the standard concentrations were also calculated using the obtained curve. The LOD and LOQ were established considering the concentrations at which the peak response a signal-to-noise ratio of at least 3 and 10 times the average noise level, respectively.

Three QC (quality control) samples with low, medium and high concentrations were used to perform the intraday and inter-day precision and accuracy. The QC concentrations were $0.2,1.5$ and $3 \mathrm{mM}$ for the analyzed sugars, respectively. Electropherograms obtained on the same day were used to determine the intra-day precision $(n=6)$ while the inter-day precision was determined on three different days $(n=18)$. Measured concentrations with target values over runs were used to determine the accuracy and were expressed as a percent of the target concentration.

\section{Nectar samples}

The validated method was applied to 210 nectar samples (B. grandiflora). The samples were collected in November 2015, from a private land adjacent to Limeburner's Creek National Park. This place is located $11 \mathrm{~km}$ north of Port Macquarie, New South Wales, Australia. All samples were stored at $-2{ }^{\circ} \mathrm{C}$ in a freezer until analysis. The collected volumes in each flower ranged between 5 to $60 \mu \mathrm{L}$. $5 \mu \mathrm{L}$ of each nectar sample were diluted $1: 40\left(\mathrm{v} \mathrm{v}^{-1}\right)$ with water $(175 \mu \mathrm{L})$ and galactose $10 \mathrm{mM}(20 \mu \mathrm{L})$ was used as IS. The samples whose concentrations were too high to fit in the calibration curve were further diluted before injection. The samples were analyzed without any sample pre-treatment.

\section{Enzymatic assay of $\alpha$-glucosidase}

$\alpha$-Glucosidase activity was assayed at $30{ }^{\circ} \mathrm{C}$ in $50 \mathrm{mM}$ citrate-phosphate, $\mathrm{pH} 5.0$ using $14 \mu \mathrm{M}$ MU $\alpha$ Glu as substrate. The reaction (performed on fluorescence microplates) was interrupted after $15 \mathrm{~h}$ by adding $0.25 \mathrm{M}$ of $\mathrm{Na}_{2} \mathrm{CO}_{3}$. The amount of 4-methylumbelliferone released was measured at $355 \mathrm{~nm}$ excitation, $460 \mathrm{~nm}$ emission and cut off $455 \mathrm{~nm} \cdot{ }^{21}$ One unit of $\alpha$-glucosidase (U) is defined as the amount of enzyme that releases $1 \mu \mathrm{mol}$ of 4-methylumbelliferone per minute.

\section{$\mathrm{pH}$ measurement in nectar samples}

One microliter of each sample was directly applied onto $\mathrm{pH}$ indicator strips Universalindikator $\mathrm{pH}$ 0-14 (Merck, Germany, Cat. No. 1.09535.0001) or Acilit ${ }^{\circledR}$ pH 0-6 (MERCK, Germany, Cat. No. 9531) and results were visually compared with the color scale.

\section{Results and Discussion}

\section{CE method development}

The best separation was obtained using an electrolyte containing $36 \mathrm{mM} \mathrm{NaOH}$ (pH ca. 12), $15 \mathrm{mM}$ sorbic acid and $0.5 \mathrm{mM} \mathrm{CTAB}$. Higher concentrations of $\mathrm{NaOH}$ did not improve the resolution of glucose and fructose peaks, and caused higher currents, resulting in excessive noise due to the Joule heating effect. Figure 1 shows a typical separation of the three target species and the IS.

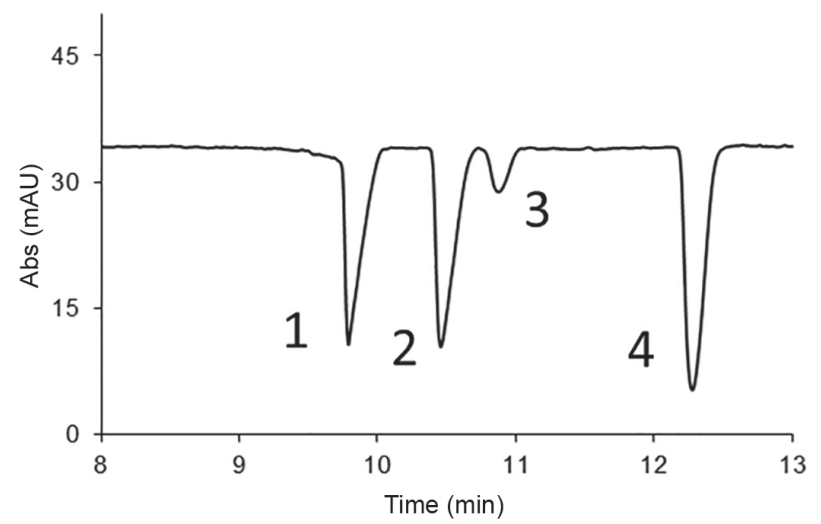

Figure 1. Example of an electropherogram of nectar sample separation: fructose (1), glucose (2), sucrose (4) and galactose-internal standard (3). Separation conditions: $36 \mathrm{mM} \mathrm{NaOH}$ at $\mathrm{pH} 12,0.5 \mathrm{mM} \mathrm{CTAB}$ and $15 \mathrm{mM}$ sorbic acid; injection, $50 \mathrm{mbar}, 8 \mathrm{~s}$; applied voltage, $-16 \mathrm{kV}$; $70 \mathrm{~cm}$ capillary, $65 \mathrm{~cm}$ effective; $25^{\circ} \mathrm{C}$; UV indirect detection. Nectar sample was diluted 1:40 before injection. 
Table 1. Analytical figures of merit for fructose, glucose, and sucrose

\begin{tabular}{|c|c|c|c|c|c|c|c|}
\hline Analyte & Linear range / $\mathrm{mM}$ & $\mathrm{R}^{2}$ & $\mathrm{LOD} / \mathrm{mM}$ & $\mathrm{LOQ} / \mathrm{mM}$ & $\begin{array}{c}\text { Precision } \\
\text { intra-day / \% }\end{array}$ & $\begin{array}{c}\text { Precision } \\
\text { inter-day / \% }\end{array}$ & Accuracy / \% \\
\hline & & & & & 3.0 & 5.5 & 99.8 \\
\hline \multirow[t]{3}{*}{ Fructose } & $0.1-4$ & 0.9940 & 0.03 & 0.1 & 2.0 & 2.5 & 97.0 \\
\hline & & & & & 2.3 & 12.0 & 103.0 \\
\hline & & & & & 4.0 & 6.0 & 99.8 \\
\hline \multirow[t]{3}{*}{ Glucose } & $0.1-4$ & 0.9925 & 0.03 & 0.1 & 3.0 & 11.0 & 98.0 \\
\hline & & & & & 7.0 & 12.0 & 100.7 \\
\hline & & & & & 5.0 & 13.0 & 98.0 \\
\hline \multirow[t]{2}{*}{ Sucrose } & $0.1-4$ & 0.9994 & 0.03 & 0.1 & 4.0 & 15.0 & 99.0 \\
\hline & & & & & 6.0 & 8.0 & 98.0 \\
\hline
\end{tabular}

$\mathrm{R}^{2}$ : coefficient of determination; LOD: limit of detection; LOQ: limit of quantification.

\section{Method validation}

The calibration curves for all sugars were linear $\left(\mathrm{R}^{2}>0.99\right)$ between 0.1 to $4 \mathrm{mM}$. All samples were diluted accordingly to fit inside the calibration curve and the peak areas were used for quantitative measurement of the analytes. Table 1 summarizes the method validation.

The LOD and LOQ were acceptable for the study purpose. The accuracy and precision at LOQ concentrations were consistent with acceptable criteria (standard deviation (SD) 20\%). ${ }^{22}$ The assays to determine the precision were performed in three days. The peak area intra-assay precision obtained was from 2 to $7 \%$. The peak area inter-assay precision ranged from 2.5 to $15 \%$. All values were within the acceptable range limits (QC low value was up to $15 \%$ and the QC medium and high values were up to $20 \%$ ). The QCs results were measured within acceptable limits of variation from 80 to $120 \%$ for the QC low and from 85 to $115 \%$ for the QC medium and high.

\section{Sugars analysis by CE}

The validated CE method was applied to 210 nectar samples. The results show that the average concentration of glucose was $0.21 \mathrm{M}$ with a minimum concentration of $0.01 \mathrm{M}$ and a maximum of $1.28 \mathrm{M}$. For fructose, the average was $0.22 \mathrm{M}$ with a minimum of $0.01 \mathrm{M}$ and a maximum of $1.40 \mathrm{M}$. The average amount of sucrose was $0.34 \mathrm{M}$ with a minimum of $0.01 \mathrm{M}$ and a maximum of $1.25 \mathrm{M}$. The average amount of sucrose found in each sample varied considerably, without any apparent trend. However, it is evident that the amounts of fructose and glucose in all individual samples were similar. Figure 2 shows the range of sugars concentration in 210 nectar samples.

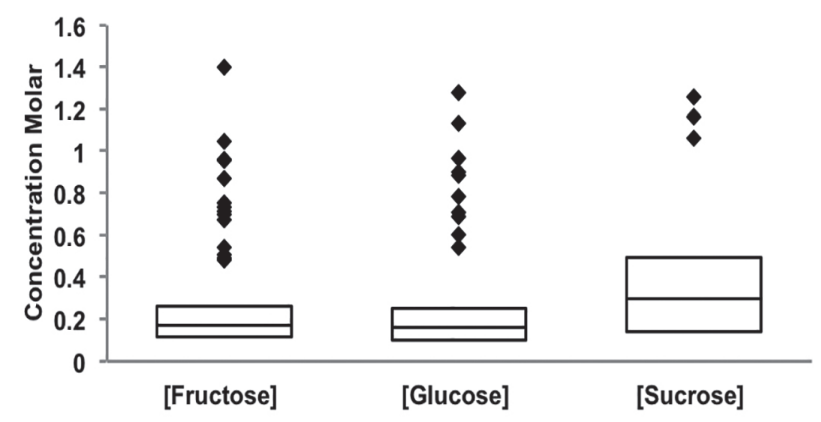

Figure 2. Box spot of the concentrations of glucose, fructose, and sucrose present in 210 nectar samples of Christmas Bellflowers.

Attempts to provide evolutionary explanations for observed nectar sugar compositions have so far been unsuccessful. It has, for example, been suggested that nectar sugar composition is related to pollinator type (e.g., bats, specialist and generalist birds, flies, bees, etc.), reflecting their different dietary preferences. However, the prevalence of sucrose, fructose and glucose in floral nectar are often inconsistent with observed dietary preferences exhibited by nectar-feeding pollinators. ${ }^{13,23-29}$

Developing such evolutionary explanations will initially require evaluation of patterns of nectar distribution, especially correlations between nectar attributes such as concentration, volume, and the sugar composition for flowers on the same plant, and this should be facilitated by the methodology presented in this article. Pollination ecologists have for many years been easily able to measure nectar volumes (e.g., with capillary tubes) and nectar sugar concentration (e.g., with hand-held refractometers) for individual flowers. ${ }^{23}$ Sugar compositions of nectar samples have been determined over about the same period, but generally limited to relatively large nectar volumes and not quick and easy to carry out. ${ }^{6}$ Now it is possible to also determine nectar sugar composition for the same individual 
flowers with a methodology that can be applied to relatively small nectar samples and is relatively quick, easy to carry out, and inexpensive.

\section{Determination of $\alpha$-glucosidase activity}

The previous observation that almost all samples contained similar amounts of glucose and fructose raised the issue of residual invertase activities in the nectar samples. Before carrying enzymatic assays, we did measure the $\mathrm{pH}$ of the nectar samples to optimize the enzymatic assays. In general, they are acidic, with pHs ranging from 3-5 $($ mean $=4.85$; median $=5)$. We performed $\alpha$-glucosidase/sucrose assays using two substrates, sucrose and 4-methylumbelliferyl- $\alpha$-D-glucopyranoside (MU $\alpha \mathrm{Glu}$ ). Using sucrose as substrate, no activity was observed (data not shown), most likely related to competitive inhibition by the excess amount of sugars in the mixtures. $\alpha$-Glucosidase activity was observed in $65 \%$ of the samples when using the fluorescent substrate MU $\alpha$ Glu. The $\alpha$-glucosidase activity in nectar samples was very low and highly variable to each sample, ranging from 0 to $0.072 \mu \mathrm{U}_{\mu} \mathrm{L}^{-1}$ with an average of $0.013 \mu \mathrm{U}_{\mu} \mathrm{L}^{-1}$. Glucosidases are ubiquitous enzymes in insects such as bees and flies and are also excreted by plants in their flowers. ${ }^{7,20}$ In this respect, the activities reported here might be of plant origin or indirectly acquired from visitor insects. Nevertheless, the presence of $\alpha$-glucosidase in the nectar is an additional factor that must be considered in further analysis of sugars from this natural source. Sucrose is the principal disaccharide found in plants phloem and nectar. This disaccharide can be enzymatically hydrolyzed in the two monosaccharides, glucose and fructose. Literature ${ }^{4}$ shows that these sugars are partly and differentially recycled through specific biochemical pathways before being secreted. It is appointed that they must be produced by sucrose breakdown after nectar secretion or from nectary cells. Invertase enzymes, such as transglucosidase and transfructosidase, contained in the secretory mechanism have the essential function of nectar chemistry and the concentration of each sugar. ${ }^{4}$

Although we have found the enzymatic activity on our samples, we could not find any correlation between this low activity and the amount of glucose or fructose present in each sample. Therefore, it can be assumed that the cleavage of sucrose producing glucose and fructose is occurring before its secretion.

\section{Conclusions}

In this article we validated a reliable CE method with indirect UV detection for the quantification of sugars in Christmas Bell nectar samples that is also suitable to other flower species. This method required minimal sample preparation without sample derivatization resulting in well-defined electropherograms with no matrix interferences. The results provided adequate LOD, reproducibility, and linearity, involving short analysis time, low consumption of reagents and low-cost. There are no great differences in sensitivity, separation time, resolution or efficiency when comparing our CE results with a similar analysis of sugars performed by $\mathrm{CE}$ or HPLC. However, the cost per sample analyzed is higher using HPLC in comparison with our CE method, which uses only a few $\mathrm{mL}$ of sodium hydroxide solution to run all those samples. The absence of any laborious derivatization procedure to analyze the samples is another advantage of our method. The method presented here did not suffer interferences of the sample matrix.

Within the same sample, glucose and fructose concentrations were similar, showing a probable activity of an $\alpha$-glucosidase. The simple approach to determine the $\mathrm{pH}$ with just one microliter of sample is simple and was essential to conduct the enzymatic assay. This activity was confirmed through the incubation of the samples with synthetic fluorescent substrates. The residual activity of this enzyme on nectar samples indicated that this or another invertase probably is acting before the sugar secretion. More studies are necessary to understand this process. In future, measurements of nectar content in the laboratory should be made with preserved samples after collection to avoid possible changes in concentration due to the occurrence of enzymatic activity. In this flower, over $70 \%$ of analyzed samples were sucrose dominant, which is consistent with bird pollination. Also, under our knowledge, this is the first time CE is applied to analyze sugars in nectar samples.

\section{Acknowledgments}

LB would like to thank the Brazilian National Council for Scientific and Technological Development-CNPq for the financial support (PROEP-ICC/CNPQ 442329/2019-9).

\section{Author Contributions}

D.M.B. performed the formal analysis and led the writing; G.P. and J.K. designed methods and collected the nectar samples in the field; L.B. and P.D. conceived the ideas, obtained the resources, perform data analysis, writing and editing equally; S.G.C.L. and F.A.G. performed the enzymatic assays and data analysis. All authors contributed critically to the drafts and final editing equally. 


\section{References}

1. Willmer, P.; Pollination and Floral Ecology; Princeton University Press: Princeton, 2011.

2. Ollerton, J.; Winfree, R.; Tarrant, S.; Oikos 2011, 120, 321.

3. Nepi, M.; J. Ecol. 2014, 102, 108.

4. Heil, M.; Trends Plant Sci. 2011, 16, 191.

5. Baker, H. G.; Baker, I. In Handbook of Experimental Pollination Biology; Jones, C. E.; John Little, R., eds.; Van Nostrand Reinhold: New York, USA, 1983, p. 117.

6. Pyke, G. H.; Nature 1991, 350, 58.

7. Kubota, M.; Tsuji, M.; Nishimoto, M.; Wongchawalit, J.; Okuyama, M.; Mori, H.; Matsui, H.; Surarit, R.; Svasti, J.; Kimura, A.; Chiba, S.; Biosci., Biotechnol., Biochem. 2004, 68, 2346.

8. Wolff, D.; Ann. Bot. 2006, 97, 767.

9. Klockow, A.; Paulus, A.; Figueiredo, V.; Amadò, R.; Widmer, H. M.; J. Chromatogr. A 1994, 680, 187.

10. Horwitz, W.; Latimer, G. W.; Official Methods of Analysis of AOAC International; AOAC International: Gaithersburg, USA, 2005, ch. 44.

11. Silva, R. F.; Tinoco, N. A. B.; Tsukui, A.; Koschnitzke, C.; Silva-Batista, I. C.; Rezende, C. M.; Bizzo, H. R.; J. Braz. Chem. Soc. 2019, 30, 388.

12. Willför, S.; Pranovich, A.; Tamminen, T.; Puls, J.; Laine, C.; Suurnäkki, A.; Saake, B.; Uotila, K.; Simolin, H.; Hemming, J.; Holmbom, B.; Ind. Crops Prod. 2009, 29, 571.

13. Lohaus, G.; Schwerdtfeger, M.; PLoS One 2014, 9, e87689.

14. Rizelio, V. M.; Tenfen, L.; da Silveira, R.; Gonzaga, L. V.; Costa, A. C. O.; Fett, R.; Talanta 2012, 93, 62.

15. Soga, T.; Food Chem. 2000, 69, 339.
16. Honda, S.; Suzuki, S.; Nitta, A.; Iwase, S.; Kakehi, K.; Methods 1992, 4, 233

17. Soga, T.; Heiger, D. N.; Anal. Biochem. 1998, 261, 73.

18. Vorndran, A. E.; Oefner, P. J.; Scherz, H.; Bonn, G. K.; Chromatographia 1992, 33, 163.

19. Zemann, A.; Nguyen, D. T.; Bonn, G.; Electrophoresis 1997, 18,1142

20. Blanes, L.; Saito, R. M.; Genta, F. A.; Donegá, J.; Terra, W. R.; Ferreira, C.; do Lago, C. L.; Anal. Biochem. 2008, 373, 99.

21. Profeta, G. S.; Pereira, J. A. S.; Costa, S. G.; Azambuja, P.; Garcia, E. S.; Moraes, C. S.; Genta, F. A.; Front. Physiol. 2017, 8,308 .

22. SWGTOX; J. Anal. Toxicol. 2013, 37, 452.

23. Hainsworth, F. R.; Wolf, L. L.; Comp. Biochem. Physiol., Part A: Mol. Integr. Physiol. 1972, 42, 359.

24. Herrera, M. L. G.; J. Mammal. 1999, 80, 683.

25. Law, B.; Aust. Mammal. 1993, 16, 17.

26. Pender, R. J.; Morden, C. W.; Paull, R. E.; Am. J. Bot. 2014, 101,201

27. Rodríguez-Peña, N.; Stoner, K. E.; Flores-Ortiz, C. M.; AyalaBerdón, J.; Munguía-Rosas, M. A.; Sánchez-Cordero, V.; Schondube, J. E.; Rev. Mex. Biodivers. 2016, 87, 465.

28. Rodríguez-Peña, N.; Stoner, K. E.; Schondube, J. E.; Ayala-Berdón, J.; Flores-Ortiz, C. M.; Martínez del Río, C.; J. Mammal. 2007, 88, 1466.

29. Stpiczyńska, M.; Nepi, M.; Zych, M.; Plant Syst. Evol. 2015, $301,1099$.

Submitted: March 17, 2020

Published online: June 16, 2020 